\title{
PROCESSO DE ENFERMAGEM EM CENTRO OBSTÉTRICO: PERSPECTIVA DOS ENFERMEIROS ${ }^{1}$
}

\author{
Tarciany Farias Fraga ${ }^{2}$, Eliane Matos ${ }^{3}$, Roberta Costa ${ }^{4}$, Nádia Chiodelli Salum ${ }^{5}$, Isabel Cristina Alves Maliska ${ }^{6}$
}

\footnotetext{
${ }^{1}$ Artigo extraído da dissertação - Processo de Enfermagem para o cuidado humanizado à parturiente de risco habitual em Centro Obstétrico, apresentada ao Programa de Pós-Graduação em Gestão do Cuidado em Enfermagem da Universidade Federal de Santa Catarina (UFSC), em 2016.

${ }^{2}$ Mestre em Gestão do Cuidado em Enfermagem. Enfermeira do Hospital Universitário Dr. Polydoro Ernani de São Thiago, UFSC. Florianópolis, Santa Catarina, Brasil. E-mail: tarcianyff@yahoo.com.br

${ }^{3}$ Doutora em Enfermagem. Enfermeira do Hospital Universitário Dr. Polydoro Ernani de São Thiago, UFSC. Florianópolis, Santa Catarina, Brasil. E-mail: elianematos@hotmail.com

${ }^{4}$ Doutora em Enfermagem. Professora do Departamento de Enfermagem, dos Programas de Pós-Graduação em Enfermagem e em Gestão do Cuidado em Enfermagem da UFSC. Florianópolis, Santa Catarina, Brasil E-mail: roberta.costa@ufsc.br

${ }^{5}$ Doutora em Enfermagem. Enfermeira do Hospital Universitário Dr. Polydoro Ernani de São Thiago, UFSC. Florianópolis, Santa Catarina, Brasil. E-mail: nchiodelli@gmail.com

${ }^{6}$ Doutora em Enfermagem. Enfermeira do Hospital Universitário Dr. Polydoro Ernani de São Thiago, UFSC. Florianópolis, Santa Catarina, Brasil. E-mail: isabel.alves07@yahoo.com.br
}

\section{RESUMO}

Objetivo: apreender a percepção de enfermeiros sobre o Processo de Enfermagem e o modelo de histórico de enfermagem realizado com a gestante/parturiente no centro obstétrico de um hospital do sul do Brasil.

Método: estudo exploratório, descritivo com abordagem qualitativa, do qual participaram treze enfermeiros que atuam em Emergência Obstétrica ou Centro Obstétrico. Os dados foram coletados no período de dezembro de 2014 a março de 2015, através de uma oficina, de entrevista individual semiestruturada e do preenchimento de um instrumento com sugestões para a elaboração de um novo modelo de histórico de enfermagem. Os dados foram analisados através da análise de conteúdo de Bardin.

Resultados: surgiram as seguintes categorias: importância e necessidade do Processo de Enfermagem; fragmentação, descontinuidade, incompletude e mecanização do Processo de Enfermagem - prejuízos à humanização; e limitações e insuficiências na abordagem e na estrutura do histórico de enfermagem - indicativos para sua reestruturação.

Conclusão: os enfermeiros do estudo compreendem que a qualidade do Processo de Enfermagem alcançará melhorias a partir de uma nova proposta de histórico, e que os diagnósticos de enfermagem, instrumentalizam e orientam o processo de cuidar.

DESCRITORES: Enfermagem obstétrica. Processos de enfermagem. Diagnóstico de enfermagem. Humanização da assistência. Gestantes.

\section{THE NURSING PROCESS IN AN OBSTETRIC CENTER: PERSPECTIVE OF NURSES}

\footnotetext{
ABSTRACT

Objective: to apprehend the nurses' perception about the Nursing Process and the nursing history model performed with the pregnant woman/parturient in the obstetric center of a hospital in southern Brazil.

Method: an exploratory, descriptive study with a qualitative approach, with the participation of thirteen nurses who work in the Obstetric Emergency or Obstetric Center. The data was collected from December 2014 to March 2015, through a workshop, a semi-structured individual interview and the completion of an instrument with suggestions for the elaboration of a new nursing history model. The data was analyzed through the Bardin's content analysis.

Results: the following categories emerged: importance and necessity of the Nursing Process; fragmentation, discontinuity, incompleteness and mechanization of the Nursing Process - damages to humanization; and limitations and shortcomings in the approach and structure of the nursing-indicative history for its restructuring.

Conclusion: the nurses of the study understand that the quality of the Nursing Process will achieve improvements based on a new historical proposal, and that the nursing diagnoses, instrumentalize and guide the care process.

DESCRIPTORS: Obstetric nursing. Nursing processes. Nursing diagnosis. Humanization of care. Pregnant women.
} 


\section{PROCESO DE ENFERMERÍA EN EL CENTRO OBSTÉTRICO: PERSPECTIVA DE LOS ENFERMEROS}

\section{RESUMEN}

Objetivo: aprehender la percepción de los enfermeros sobre el Proceso de Enfermería y el modelo histórico de la enfermería realizados con la gestante/parturienta en el centro obstétrico de un hospital do sur del Brasil.

Método: estudio exploratorio, descriptivo y con abordaje cualitativo en el que participaron trece enfermeros que actúan en la Emergencia Obstétrica o Centro Obstétrico. Los datos fueron obtenidos entre Diciembre del 2014 y Marzo del 2015 a través de un taller de entrevista individual semiestructurada y del relleno de un instrumento con sugestiones para la elaboración de un nuevo modelo del histórico en enfermería. Los datos fueron analizados a través del análisis del contenido de Bardin.

Resultados: surgieron las siguientes categorías: importancia y necesidad del Proceso de Enfermería, fragmentación, discontinuidad, incompletitud y mecanización del Proceso de Enfermería, perjuicios para la humanización, limitaciones e insuficiencias en el abordaje y estructura del histórico de enfermería e indicativos para su reestructuración.

Conclusión: los enfermeros del estudio comprenden que la cualidad del Proceso de Enfermería alcanzará mejorías a partir de una nueva propuesta del histórico, y también, los diagnósticos de enfermería instrumentalizan y orientan el proceso de cuidar.

DESCRIPTORES: Enfermería obstétrica. Procesos de enfermería. Diagnóstico de enfermería. Humanización de la asistencia. Gestantes.

\section{INTRODUÇÃO}

A gestante parturiente recebe um cuidado de qualidade quando lhe é permitida uma experiência positiva através da manutenção da sua saúde física e emocional, do respeito a sua integralidade e do acompanhamento contínuo a fim de prevenir e resolver as intercorrências que possam surgir.

Os profissionais de saúde são grandes colaboradores dessa experiência da mulher, pois desempenham importante papel neste momento vital ao atuarem com competência. Ao colocarem o seu conhecimento à disposição da manutenção do equilíbrio físico/psíquico da gestante e do recémnascido, podem reconhecer os momentos críticos em que suas intervenções são necessárias para assegurar o bem-estar de ambos. ${ }^{1}$

O enfermeiro participa da equipe multiprofissional e desempenha importante papel no cuidado prestado à gestante. Por meio do Processo de Enfermagem (PE) o enfermeiro integraliza a assistência de enfermagem à parturiente, planejando e promovendo um cuidado específico conforme suas necessidades. A enfermagem tem oportunidade de criar vínculo com a parturiente e promover um cuidado diferenciado e efetivo à mesma ${ }^{1}$, através de uma assistência qualificada, acolhedora e humanizada.

Conforme a Lei $\mathrm{n}^{\mathrm{o}} 7.498$, de 25 de junho de 1986, que dispõe sobre a regulamentação do exercício da Enfermagem, cabe ao enfermeiro, como integrante da equipe: a assistência de enfermagem à gestante, à parturiente e à puérpera; o acompanhamento da evolução do trabalho de parto e parto; e a execução do parto sem distócia. ${ }^{2} \mathrm{O}$ Conselho Federal de Enfermagem (COFEN), por meio da Resolução 358/2009, dispõe, ainda, sobre a Sistematização da Assistência de Enfermagem (SAE) e descreve que a implementação do PE deve ser realizada em todas as instituições brasileiras onde o enfermeiro presta cuidados de enfermagem, sendo uma função privativa do mesmo. ${ }^{2}$

Portanto o PE é um instrumento metodológico que orienta a assistência e a documentação da atuação da enfermagem, oferecendo maior visibilidade e reconhecimento profissional. ${ }^{2} \mathrm{O}$ mesmo ainda oportuniza a valorização das ações dos profissionais, a delimitação das suas competências e a conquista dos seus espaços. ${ }^{3}$

Apesar de toda a importância do PE para a assistência, muitos fatores dificultam a sua execução, como a falta de conhecimento e conscientização para a sua realização, além de questões relacionadas à infraestrutura, déficit de pessoal, sobrecarga de trabalho, falta de apoio da chefia e gestores, falta de recursos materiais, entre outros. ${ }^{4}$

O PE é cíclico, dinâmico e apresenta, de acordo com a maioria dos autores, de cinco a seis etapas, iniciando pelo histórico de enfermagem, seguido das etapas de identificação dos diagnósticos de enfermagem, da definição do plano assistencial, da prescrição de enfermagem, da evolução de enfermagem e do prognóstico. ${ }^{5}$

A primeira etapa do $\mathrm{PE}$, o histórico de enfermagem, tem a finalidade de coletar informações sobre o indivíduo, sua família e sua comunidade., ${ }^{2,5}$ Portanto, a sua adequada estrutura é importante para a continuidade das demais etapas do PE.

A coleta de dados para a enfermagem precisa ser abrangente de modo que origine dados que orientem a assistência de enfermagem para a promoção e proteção da saúde, que gerem diagnósticos de enfermagem, que corresponde à segunda etapa do $\mathrm{PE}$, para assim dar continuidade às próximas 
etapas. Vale enfatizar que os diagnósticos de enfermagem são interpretações de dados levantados, que orientam o planejamento, a implementação e a avaliação dos enfermeiros. ${ }^{6}$

Para que o PE seja realizado de forma efetiva, é necessário o envolvimento dos enfermeiros assistenciais, a fim de realizarem um histórico de enfermagem completo que possibilite visualizar as necessidades das mulheres e prescreverem cuidados individualizados em cada situação.

Assim, este estudo teve por objetivo apreender a percepção de enfermeiros sobre o Processo de Enfermagem e sobre o modelo de histórico de enfermagem realizado com a gestante/parturiente no centro obstétrico de um hospital do Sul do Brasil.

\section{MÉTODO}

Trata-se de um estudo exploratório, descritivo, com abordagem qualitativa, realizado na maternidade de um hospital universitário do Sul do Brasil. No hospital em estudo, o PE é realizado com base na Teoria das Necessidades Humanas Básicas adaptado por Wanda Aguiar Horta. O PE à gestante se inicia com a realização do histórico de enfermagem pelo enfermeiro da emergência obstétrica, no momento da internação, antes da mesma ser encaminhada ao centro obstétrico. O enfermeiro do centro obstétrico realiza a prescrição de enfermagem por meio de um impresso previamente elaborado, em que consta uma relação tipo checklist dos cuidados mais frequentes necessários à gestante/parturiente. A prescrição baseia-se na identificação de "problemas de enfermagem", conforme propõe o modelo de Wanda de Aguiar Horta. ${ }^{5}$ A etapa do diagnóstico de enfermagem não é realizada.

Considerando esse contexto, foram convidados a participarem do estudo, os nove enfermeiros da emergência obstétrica e os nove do centro obstétrico, além dos três residentes da área da saúde da mulher que desenvolviam atividades nestes setores. $\mathrm{O}$ critério de participação foi atuar na unidade há pelo menos seis meses e foram excluídos os enfermeiros que estavam de férias e/ ou afastados do trabalho no momento da coleta de dados. A amostra do estudo foi composta por 13 enfermeiros, sendo quatro do centro obstétrico, seis da emergência obstétrica e três da Residência Integrada Multiprofissional em Saúde na Atenção em Saúde da Mulher e da Criança. Nenhum profissional se recusou a participar da pesquisa.

A coleta de dados foi realizada no período de dezembro de 2014 a março de 2015. Inicialmente, foi realizada uma oficina com os enfermeiros para mobilização dos mesmos, reflexão sobre o PE e reestruturação do histórico de enfermagem da parturiente utilizado na maternidade. Participaram desta oficina apenas três enfermeiros. Neste encontro, foi desenvolvida uma exposição dialogada sobre o Processo de Enfermagem e a importância da etapa de diagnóstico, por uma enfermeira especialista na temática. Em seguida, a proposta foi avaliar o modelo de histórico de enfermagem utilizado na instituição. Além do modelo de histórico, foi fornecido um impresso para os participantes apontarem os pontos fortes, fragilidades e sugestões, e um impresso com as Necessidades Humanas Básicas, para avaliarem a necessidade de inclusão de alguma necessidade humana básica no modelo atual de histórico de enfermagem. Contudo, devido ao reduzido número de participantes, foi sugerido que esta atividade fosse desenvolvida de forma individual e que também fossem convidados os demais enfermeiros dos setores envolvidos para contribuir com a proposta.

Em seguida, foi realizada uma entrevista individual semiestruturada com os enfermeiros e residentes de enfermagem do centro obstétrico e da emergência obstétrica para conhecer, com mais detalhes, sua percepção acerca do PE atualmente realizado à parturiente na instituição do estudo. As entrevistas foram gravadas com o consentimento dos participantes. Conforme a escolha dos participantes, as entrevistas foram realizadas na própria instituição e duraram de trinta minutos a uma hora e vinte minutos. Todas foram transcritas e juntamente com os instrumentos propostos para a oficina serviram de dados para análise dos resultados desta pesquisa.

A análise dos dados seguiu as etapas propostas por Bardin: ${ }^{7}$ pré-análise, onde foi realizada a organização e leitura cuidadosa de todo material; exploração do material a partir da leitura horizontal dos dados em seu conjunto, buscando neste processo as aproximações entre as falas; análise da temática, que foi dividir o texto por aproximação e similaridade em temas principais; e tratamento das informações, inferência e a interpretação, quando as categorias que foram utilizadas como unidades de análise são analisadas à luz da literatura atual. ${ }^{7}$

As categorias e subcategorias que surgiram na análise dos dados foram as seguintes: importância e necessidade do Processo de Enfermagem; fragmentação, descontinuidade, incompletude e mecanização do Processo de Enfermagem - prejuízos à humanização; e limitações e insuficiências na 
abordagem e na estrutura do histórico de enfermagem - indicativos para sua reestruturação.

A pesquisa foi submetida ao Comitê de Ética em Pesquisa da UFSC, sendo direcionado pela Plataforma Brasil, ao Hospital Infantil Joana de Gusmão, que emitiu o Certificado de Apresentação para Apreciação Ética (CAAE) no 37860814.1.0000.5361.

A pesquisa foi norteada pelas diretrizes e normas regulamentadoras da Resolução n. 466/2012. Os participantes foram identificados pela letra " $\mathrm{E}$ ", que se refere a enfermeiro, seguida de um número correspondente para preservar a identidade.

\section{RESULTADOS}

Os resultados estão apresentados de modo a permitir um entendimento das experiências e expectativas desses profissionais a respeito do $\mathrm{PE}$ conforme o vivenciam em seu contexto de trabalho, e são apresentados a seguir.

\section{Importância e necessidade do Processo de Enfermagem}

Os participantes reconhecem a importância da utilização do PE, inclusive da etapa de histórico, sendo que o veem como possibilidade de melhorar a qualidade da assistência prestada às gestantes/ parturientes, estando voltado às necessidades individuais, através de um método que direciona o processo de cuidar, conforme expressam nos depoimentos abaixo: o Processo de Enfermagem é muito importante, ele facilita o processo de cuidado às mulheres (E1). É necessário para manter a assistência de enfermagem com qualidade [...] (E2). Eu acho o histórico de enfermagem extremamente importante, pois direciona o cuidado da enfermagem à mulher e possibilita conhecê-la como um todo [...] (E7).

$\mathrm{O} \mathrm{PE}$, além de promover qualidade à assistência, também contribui para o fortalecimento da enfermagem como ciência, gerando autonomia, satisfação e reconhecimento profissional. Portanto, é necessário que os enfermeiros empenhem-se em manter essa conquista. O relato expressa essa constatação: eu acho que o Processo de Enfermagem empodera a profissão, eu acho que é um quesito que a gente tem que bater na tecla [...] (E6).

Apesar de os participantes realçarem a importância e a necessidade da implementação do PE no cuidado às gestantes/ parturientes, também destacaram as fragilidades que vivenciam no contexto de sua atuação.
Fragmentação, descontinuidade, incompletude e mecanização do Processo de Enfermagem -prejuízos à humanização

Na instituição estudada, o PE realizado à gestante/ parturiente começa com a aplicação do histórico de enfermagem pelo enfermeiro da emergência obstétrica no momento da internação, antes da paciente ser encaminhada ao centro obstétrico. No centro obstétrico, o enfermeiro que a recebe realiza a prescrição de enfermagem, essa prescrição é um impresso tipo checklist que apresenta a relação dos cuidados mais frequentes à gestante/parturiente a serem prescritos.

Segundo os participantes, os enfermeiros muitas vezes desconsideram os dados do histórico de enfermagem ao realizarem a prescrição de enfermagem, pois consideram o mesmo incompleto e percebem que faltam dados importantes sobre a gestante/parturiente em sua estrutura. O hospital em estudo não trabalha com diagnóstico de enfermagem e no centro obstétrico não é realizada a evolução de enfermagem. Essa última etapa é realizada habitualmente pelos enfermeiros da unidade de alojamento conjunto, após a transferência da parturiente para esta unidade.

Sendo assim, o PE, do modo como está instituído, limita o cuidado à rotina da unidade e à previsão de cuidado de forma individualizada, como também, muitas vezes, não evidencia cuidados que são implementados, mas não registrados. As falas refletem esta conjuntura: o Processo de Enfermagem, que é realizado aqui nas pacientes da maternidade, é feito em etapas, só que são etapas fragmentadas porque na Emergência Obstétrica, por exemplo, é feito só o histórico de enfermagem, depois a paciente entra para o Centro Obstétrico. No Centro Obstétrico não é feito evolução de enfermagem, também não é trabalhado o diagnóstico de enfermagem [...] (E4). [...] é um histórico de enfermagem que eu não consigo enxergar a mulher, a gestante e parturiente como ela está. Não consigo ver esta mulher num todo, eu a vejo em partes. Eu não consigo avaliar como esta mulher está chegando ao Centro Obstétrico porque está faltando dados [...] (E3). A prescrição de enfermagem, em minha opinião, é muito geral [...] não é individualizada (E7). Durante o trabalho de parto, que às vezes dura o dia inteiro, o enfermeiro faz muitas coisas, então, se fosse registrado [...] acho que seria bem interessante fazer a evolução (E7).

Os participantes deixam transparecer em suas falas que o PE instituído no seu contexto de trabalho é fragmentado e apresenta lacunas, ou seja, o fluxo de suas etapas apresenta limitações para acontecer de forma inter-relacionada e interdependente. 
Os resultados apontam um PE realizado de forma tecnicista ao considerarem que o histórico de enfermagem é realizado de forma automatizada e rápida, que a prescrição é extremamente padronizada e não individualizada. Vejo que está sendo aplicado de forma automatizada, estamos perdendo a visão humanizada e muito mais, realizando por obrigação. O histórico de enfermagem na Emergência Obstétrica é robotizado (E2). Eu acho que a prescrição de enfermagem é extremamente padronizada, isso é muito ruim porque tem particularidades em cada mulher que às vezes não é levado em conta (E3). A prescrição de enfermagem [...] não é individualizada [...] isso acaba mecanizando o cuidado. [...] acaba não vendo as particularidades de cada mulher (E7).

Percebe-se nas falas dos participantes que o PE realizado não coincide com a perspectiva da individualização do cuidado, interferindo nos princípios da humanização do parto e puerpério. Predominam no relato dos participantes palavras como: automatizado, robotizado, congelado, pronto, mecanizado.

\section{Limitações e insuficiências na abordagem e na estrutura do histórico de enfermagem - indicativos para sua reestruturação}

Especificamente, em relação ao histórico de enfermagem que deveria subsidiar a elaboração das demais etapas do PE, os participantes entendem que o mesmo não cumpre esse papel, repete informações existentes no histórico médico, ou seja, relatam que há problemas em sua estrutura.

Eu acho que ele tem que ser mais objetivo porque a maioria das mulheres já chega num processo de dor (E8). Urge uma mudança no histórico para retirar as repetições de dados, excluir dados da anamnese coletados e descritos pela medicina que simplesmente são copiados pela enfermagem. Alguns problemas físicos eu consigo identificar. Os de ordem psicoemocional e relacional não (E2). Acho que deveria focar mais nas coisas que nos interessa para planejar o cuidado de enfermagem propriamente dito (E3).

Acho que poderia ter questões também que abordassem mais as informações das mulheres quanto ao pré-natal e sobre trabalho de parto e nascimento, quanto ela já conhece disso, quanto precisa ser orientada no centro obstétrico. [...] é importante a gente saber disso também pra planejar a assistência (E9). Tem que dar abertura pra mulher falar o que ela espera em relação ao parto [...] (E10). Talvez colocar mais dados dos partos anteriores, por exemplo, cesárea, qual o motivo que levou aquela cesárea, qual a expectativa da mulher quanto a isso, como foi pra ela ter uma cesárea anterior ou como foi o parto pra ela [...] também deveria colocar a questão do acompanhante (E7). O nosso histórico tem que perder um pouquinho da formatação checklist [...] sobre a dor não sei se não poderia ter uma escalinha de dor, de 1 a 10, a tua dor é tanto (E13).

As falas destacadas apresentam sugestões de mudança no histórico de enfermagem utilizado, atualmente, na maternidade da instituição tanto no que diz respeito à estrutura quanto à priorização na abordagem de aspectos mais significativos da assistência de enfermagem. Relatam a ideia de incluir e aprofundar no histórico de enfermagem questões relacionadas aos aspectos psicossociais, à percepção dolorosa, se recebeu informações e orientações no pré-natal, expectativas e dúvidas sobre a internação, sobre o trabalho de parto e parto, seus medos, entre outros, assim como defendem a inclusão de questões abertas no instrumento, com mais espaço para registros mais livres.

Por outro lado, percebem-se nos relatos oposições relacionadas à configuração mais ampliada do histórico de enfermagem. Alguns participantes entendem que o mesmo deve ser mais objetivo, que deve deter-se unicamente às questões da assistência de enfermagem.

Os indicativos para a reestruturação do atual histórico de enfermagem resultam de todas as etapas do estudo, considerando as sugestões apresentadas pelos participantes durante a oficina e durante as entrevistas. As falas dos participantes sugerem a atualização de todas as etapas do PE aplicado às gestantes/parturientes internadas, assim como a necessidade de implementação destas no cuidado à gestante/parturiente que é atendida no centro obstétrico.

Dentre as sugestões, destaca-se a reestruturação do histórico de enfermagem e a inclusão da etapa do diagnóstico de enfermagem, das prescrições realizadas com base nos diagnósticos de enfermagem de cada gestante/parturiente na emergência obstétrica. No centro obstétrico, ressalta-se a realização da etapa da evolução e a informatização do PE em sua totalidade. Sugere-se, ainda, a manutenção da fundamentação do PE pela Teoria das Necessidades Humanas Básicas, conforme é adotada atualmente na instituição. Implícita nestas falas está a necessidade de discussão coletiva para provocar as alterações necessárias neste instrumento de trabalho da enfermagem: é que o Processo de Enfermagem tem algumas falhas que precisam ser corrigidas e até melhoradas. Essa discussão do Processo de Enfermagem é importante para avaliarmos como estamos fazendo esse processo aqui no exercício da nossa profissão como enfermeiros (E5). É muito importante incluir o diagnóstico de enfermagem, 
por mim já estaria incorporado no sistema informatizado do HU [...] temos que entender essa etapa como algo que realmente muda o cuidado, principalmente quando se fala de um hospital que é referência e de um hospital que tem o centro obstétrico que é considerado humanizado. [...] temos que ver o diagnóstico de enfermagem como algo que vai proporcionar àquela mulher um cuidado que é só dela, nenhuma outra paciente vai ter o mesmo cuidado [...] (E3). Primeiro precisa realmente melhorar o histórico de enfermagem para depois implementar o diagnóstico de enfermagem (E9). Baseado no diagnóstico, temos que montar uma nova prescrição, para ser mais individualizada (E3). Não fazemos a evolução de enfermagem [...] a evolução tem que começar [...] (E10).

Outro aspecto abordado como necessário para repensar o histórico de enfermagem é a realização de capacitação para os enfermeiros assegurando, deste modo, a realização do processo com qualidade.

Sobre a necessidade de capacitação, identificase nas entrevistas que a maioria dos participantes conhece a classificação diagnóstica de enfermagem da NANDA, que apenas dois conhecem a Classificação Internacional para a Prática de Enfermagem (CIPE) e que três não conhecem nenhuma classificação diagnóstica de enfermagem, corroborando com a percepção dos mesmos acerca da importância de processo de capacitação para a prática assistencial. Esse processo envolve tanto a questão da sistematização da assistência de enfermagem como a especificidade do próprio trabalho da maternidade e da sua clientela: eu vejo uma diferença das enfermeiras que têm alguma experiência em centro obstétrico daquelas que não fazem nem ideia de como funciona o trabalho de parto. Eu vejo que eu preencho o histórico de uma maneira diferente, porque a gente sabe o que a paciente vai ter aqui dentro, a gente conhece como flui o trabalho de parto [...]. A enfermeira que deveria preencher esse histórico, deveria ser obrigatoriamente alguém que tivesse conhecimentos obstétricos (E3). Nós enfermeiras, pouco sabemos fazer o diagnóstico de enfermagem. Falta-nos capacitação contínua e supervisão por parte das chefias. Capacitação continuada em conjunto para que tenhamos uma mesma linguagem, sincronização, confiança e flexibilização (E2).

Outro aspecto muito discutido entre os participantes é a questão do dimensionamento de pessoal, em especial, o número adequado de enfermeiros para a realização do processo: então esse é um setor, que muitas vezes, exige que tivesse duas enfermeiras, com uma enfermeira cuidando de quem está chegando e a outra cuidando da outra parte da assistência, então assim, o fato de ter uma enfermeira compromete a aplicação do Processo de Enfermagem (E11).
Contudo, os participantes deixam claro que a melhoria do PE não se limita à capacitação dos profissionais e à quantidade de profissionais para implementá-lo. É necessário repensar e reestruturar todo o PE, ou seja, realizar uma ampla mudança. Nesta perspectiva, discutem o que poderia ser mantido, uma vez que atende as necessidades, e o que poderia ser modificado.

Como aspectos a serem mantidos, estão: a teoria de enfermagem da Wanda de Aguiar Horta, que possibilita ao enfermeiro usar um método científico; o esquema de preenchimento com questões de múltipla escolha; tamanho da fonte; e manutenção de informações importantes para a continuidade do cuidado.

Como aspectos a serem modificados ou melhorados, os participantes citam: espaço para descrever dados sobre a gestante/parturiente que o instrumento não contempla; tornar o instrumento mais sucinto; rever a manutenção de itens que dificilmente são preenchidos; rever itens que estão desatualizados; rever a orientação do mesmo que privilegia mais as questões físicas e faz pouca abordagem das necessidades psicossociais e emocionais; acrescentar informações importantes; rever e redefinir os dados que poderiam ser preenchidos no centro obstétrico; promover a informatização de todo o processo; criar espaço e condições para que o enfermeiro apresente os diagnósticos de enfermagem e planeje um cuidado individualizado; trabalhar coletivamente a reflexão acerca do processo e focar o histórico nas necessidades imediatas da gestante.

Acrescentam, ainda: incluir e aprofundar mais as questões psicossociais; acrescentar a avaliação de dor/desconforto e aprofundar mais esta questão; dar espaço para a mulher falar a respeito dos seus conhecimentos e dúvidas sobre o trabalho de parto e parto e outros assuntos; permitir informações mais amplas e individualizadas da gestante/parturiente; levantar questões relacionadas às expectativas com relação à internação e ao tipo de parto; incluir um item que identifique se a gestante tem um plano de parto; incluir informações relacionadas à apresentação de comorbidades prévias e doenças gestacionais.

\section{DISCUSSÃO}

Dos resultados do estudo, confirma-se entre todos os participantes a compreensão quanto à valorização do PE no processo do parto e nascimento. Suas falas apresentam a compreensão de que o PE possibilita a apresentação das necessidades da parturiente, o planejamento e a implementação do cui- 
dado a esta. Ele norteia o raciocínio do enfermeiro no planejamento da assistência, sendo considerado um processo decisório. ${ }^{8}$

Vale ressaltar, também, que o PE favorece segurança nas decisões, diminui a fragmentação e garante a continuidade do cuidado, além de proporcionar visibilidade ao trabalho realizado, podendo contribuir para a consolidação da enfermagem como ciência.9-

Os participantes afirmam que os registros de enfermagem favorecem a comunicação e a continuidade do cuidado, e reforçam em suas falas que a carência de registros de dados relevantes sobre a gestante/parturiente no histórico de enfermagem e dos cuidados de enfermagem, influencia na assistência prestada. De igual forma, estudos afirmam que os registros incompletos no histórico e plano de cuidados, pode interferir negativamente na qualidade da assistência. ${ }^{10-13}$

Referindo-se às fragilidades e aos desafios do $\mathrm{PE}$, destaca-se nas falas dos participantes a fragmentação deste ao longo do trajeto da gestante/parturiente/puérpera na instituição. Embora o processo tenha sido idealizado para ocorrer em um contínuo em que cada etapa efetuada anteriormente fosse avaliada e integrada à fase seguinte, isso não acontece. $\mathrm{Na}$ atualidade, por cada espaço em que circula esta mulher - emergência obstétrica, centro obstétrico e alojamento conjunto - desenrola-se de modo um tanto independente as diversas fases do PE. Essa fragmentação é nociva à humanização e à individualização do cuidado, assim como à segurança da mulher.

Conforme destacado por Horta e pela Resolução 358/2009, as fases do PE (histórico de enfermagem; diagnóstico de enfermagem; planejamento de enfermagem; implementação; e avaliação de enfermagem) devem ser organizadas de forma inter-relacionadas, interdependentes e recorrentes. ${ }^{2,5}$

É notável que os participantes desejem realizar todas as etapas do PE, porém há fatores que dificultam esta prática. Existem lacunas como a ausência de algumas etapas, os instrumentos que não promovem a adequada continuidade à próxima fase, gerando a falta de dinamismo e pouca inter-relação entre suas etapas.

Há limitações na estrutura do histórico de enfermagem utilizado, que fazem com que os participantes entendam como necessária a retirada de determinados dados, qualificando-os como abordagem médica. No entanto, é necessário avaliar se estes são essenciais ou não ao planejamento da assistência de enfermagem, pois qualquer dado do indivíduo corresponde a sua particularidade e o enfermeiro precisa conhecê-lo. A enfermagem, como uma profissão que colabora com muitas disciplinas de saúde, por vezes, coleta e compartilha informações médicas, dados farmacêuticos e outras informações sobre o indivíduo, pois todos os dados têm um propósito para quem está tomando uma decisão sobre os cuidados do mesmo. ${ }^{14}$

O histórico de enfermagem é um roteiro sistematizado para o levantamento dos dados do ser humano, significativos para o enfermeiro, que tornam possível a identificação de seus problemas ${ }^{5}$ e, consequentemente, a apresentação de diagnósticos de enfermagem. Na instituição investigada, o modelo de histórico de enfermagem existente, em sua formatação atual, dificulta a identificação e visualização dos problemas de enfermagem e a elaboração de diagnósticos de enfermagem. Assim, se esta etapa é realizada de forma inadequada, ou não é realizada, as demais etapas do PE ficam prejudicadas. ${ }^{15}$

Com relação à forma como o instrumento histórico de enfermagem é utilizado, a experiência do enfermeiro na coleta de dados é indispensável ao sucesso da atividade, assim, quanto maior seu tempo na execução, maior será a sua habilidade e menor o tempo gasto nesta atividade. ${ }^{5}$ Cabe enfatizar, que questionamentos profundos não fazem parte da organização de coleta de dados, porque não há como incluir todos os prováveis questionamentos que possam ser feitos em relação a cada possibilidade de resposta humana. ${ }^{14}$ Portanto, é através do conhecimento científico e do conjunto de experiências que o enfermeiro vai se habilitando cada vez mais para a realização do mesmo.

O diagnóstico de enfermagem é um dos elementos importantes no processo de cuidar, pois dele surgem todos os cuidados do plano assistencial. Ainda estabelece metas, condutas e a realização da avaliação da assistência de enfermagem prestada. ${ }^{16}$ Ele é considerado a interpretação científica dos dados coletados do indivíduo, conduz o planejamento e a implementação das intervenções para melhores resultados. Outros autores reforçam este pensamento quando relatam que o mesmo, considerando as necessidades e segurança do indivíduo, se constitui em elemento essencial para a assistência de enfermagem, baseado em evidências. ${ }^{6}$

O PE concomitante com a visão holística do enfermeiro favorece uma assistência individualizada à parturiente e baseada no conhecimento científico, oportunizando-lhe sentir-se parte de um processo natural, acompanhando o ritmo do seu próprio corpo. ${ }^{17}$ 
Para humanizar, através da individualidade e integralidade do cuidado, o enfermeiro precisa desenvolver ações diferenciadas que superem o modelo tecnicista e mecanicista. É necessário que durante a implementação do $\mathrm{PE}$, os indivíduos tenham espaço para falar e tirar suas dúvidas. Pois é uma das propriedades do PE viabilizar o vínculo entre enfermeiro e usuário desenvolvendo relações humanizadas. $^{8}$

Humanização compreende um olhar amplo para as necessidades de saúde dos indivíduos, considerando os aspectos biopsicossociais no seu contexto de vida. ${ }^{18}$ Portanto, não há PE de qualidade se não há humanização, porque o PE deve ser estruturado e implementado de forma que se consiga identificar e assistir globalmente as necessidades do indivíduo.

É evidente que todo o trabalho realizado em uma instituição precisa de profissionais capacitados e recursos humanos adequados para realizá-lo. Entretanto, referindo-se ao PE, os enfermeiros devem ser preparados não só para que sejam capazes de executar técnicas de trabalho, mas também, para que sejam criativos e que reflitam sua prática, para que tenham autonomia e capacidade de resolver problemas, e para que sejam comprometidos com a ética e a transformação da realidade. ${ }^{19}$

Para introduzir o PE, é necessário que a quantidade suficiente de recursos humanos seja uma das prioridades dos gestores dos serviços de saúde, ${ }^{20}$ ou seja, o dimensionamento de enfermeiros é uma questão essencial para a sua implementação. ${ }^{21}$

A presença e atuação dos gestores é grandiosamente importante em todos os processos de trabalho que acontecem em qualquer instituição, mas vale destacar, mais especificamente em relação ao $\mathrm{PE}$, que os enfermeiros podem desenvolvê-lo sem esperar que seja determinada a sua execução, ${ }^{9}$ ou seja, os enfermeiros podem iniciar coletivamente um processo de atualização do PE em sua prática.

Os obstáculos na realização do PE, citados na literatura e presentes nos resultados deste estudo, estão relacionados com fatores de sua própria organização e estrutura, com a falta de dinamismo e inter-relacionamento entre as fases, com a deficiência de um ensino mais eficaz acerca das técnicas do exame físico e das teorias que o fundamentam. Muitas vezes, também a falta de recursos humanos e materiais, entre outros, interfere neste processo. ${ }^{22}$

A implantação da SAE é uma exigência para todas as instituições de saúde do Brasil, tanto públicas como privadas. ${ }^{2}$ No entanto, no cotidiano da prática profissional, visualiza-se que o PE ainda não está totalmente implantado nos serviços de saúde, como também muitas dificuldades são encontradas em sua consolidação. ${ }^{8} \mathrm{O}$ dimensionamento inadequado de profissionais, a escassez de programas de educação permanente e a falta de motivação e envolvimento das equipes são apontados como fatores que dificultam a implementação da SAE. ${ }^{20}$

No entanto, este instrumento é fundamental para garantir um cuidado baseado em evidências científicas e com qualidade à mulher e ao seu filho, durante o trabalho de parto e parto, assim como para a segurança da equipe de enfermagem. O PE é considerado um documento legal, pois com suas etapas registradas no prontuário do cliente a equipe de enfermagem fica respaldada. ${ }^{8}$

Acredita-se que uma das estratégias que potencializam o uso do PE é a sua informatização. Atualmente, as aplicações eletrônicas dão suporte a muitas atividades diárias, e os enfermeiros esperam que o sistema clínico de informações forneça suporte na realização do PE. ${ }^{14}$ Esse dado vai ao encontro do pensamento da maioria dos participantes que demonstra acreditar nessa estratégia ao enfatizar a necessidade da inclusão do PE, realizado no Centro Obstétrico, no sistema informatizado da instituição.

Por fim como limitações deste estudo, destacam-se a escassez de materiais publicados em relação a SAE tanto nacional quanto internacionalmente, e a dificuldade em reunir os participantes para a realização de oficinas, o que poderia promover uma maior discussão sobre a temática.

\section{CONCLUSÃO}

Os participantes reconhecem a relevância do $\mathrm{PE}$ em diversos benefícios relacionados à gestante/ parturiente, à profissão e à equipe de enfermagem. Consideram o PE um método sistematizado composto pela união da teoria com a prática que, por meio do raciocínio clínico do enfermeiro, norteia uma assistência de enfermagem segura, voltada para satisfazer as necessidades individuais de cada gestante/parturiente; favorece o registro das ações e das orientações no prontuário, ou seja, todo o processo assistencial fica documentado; promove satisfação profissional, reconhecimento e visibilidade da profissão; proporciona o vínculo entre o enfermeiro e a gestante/parturiente; e facilita a continuidade da assistência.

As limitações referidas pelos participantes decorrem da reflexão acerca da prática e apontam aspectos a serem aprimorados com vistas à assistência de qualidade à gestante/parturiente. Destaca-se, nestas 
sugestões, a necessidade de capacitação permanente, o adequado incremento de recursos humanos para a qualidade da assistência e, ainda, o indicativo de informatização do processo como um todo.

É urgente a reflexão, discussão e definição do andamento deste processo entre os enfermeiros da emergência obstétrica e do centro obstétrico, pois o modo como está implementado o PE pode interferir positiva ou negativamente na assistência de qualidade. Espera-se que este estudo possa contribuir para fomentar o debate sobre a implementação do $\mathrm{PE}$, no âmbito do cuidado à gestante/parturiente, junto aos enfermeiros que atuam nas diversas instituições do país e que enfrentam dificuldades na operacionalização das etapas do mesmo.

\section{REFERÊNCIAS}

1. Gramacho RCCV, Silva RCV. Enfermagem na cena do parto. In: Brasil, Ministério da Saúde. Universidade Estadual do Ceará. Cadernos HumanizaSUS: Humanização do parto e nascimento. v. 4. Brasília (DF): Ministério da Saúde, 2014.

2. Conselho Regional de Enfermagem. Série Cadernos Enfermagem: consolidação da legislação e ética profissional. 2 ed. Florianópolis (SC): Quorum Comunicação, 2013.

3. Pivoto FL, Lunardi Filho WD, Lunardi VL, Silva PA, Busanello J. Produção de subjetividade do enfermeiro: relação com a implementação do processo de enfermagem. Rev Enferma UFPE on line. [Internet]. 2017 [cited 2017 May 20]; 11(4):1650-7. Available from: https:/ / periodicos.ufpe.br/revistas/revistaenfermagem/article/ viewFile/15261/18057

4. Takahashi AA, Barros ALBL, Michel JLM, Souza MF. Dificuldades e facilidades apontadas por enfermeiras de um hospital de ensino na execução do processo de enfermagem. Acta Paul Enferm [Internet]. 2008 [cited 2017 May 22]; 21(1):32-8. Available from: http://www. scielo.br / scielo.php?script=sci_arttext\&pid=S010321002008000100005\&lng=en

5. Horta WA. Processo de enfermagem. Rio de janeiro: Guanabara Koogan, 2011.

6. Lunney M. Coleta de dados, julgamento clínico e diagnósticos de enfermagem: como determinar diagnósticos precisos. In: Nanda-I. Diagnósticos de enfermagem da Nanda International: definições e classificações: 20122014. Porto Alegre (RS): Artmed, 2013.

7. Bardin L. Análise de conteúdo. São Paulo: Edições 70,2011.

8. Medeiros AL, Abrantes RM, Santos SR, Nóbrega MML. Sistematização da assistência de enfermagem como um processo de trabalho da enfermagem: uma reflexão crítica. Rev Enferm UFPE on line. [Internet] 2010 [cited 2016 Set 07]; 4(3):1571-6. Available from: http://www.revista.ufpe.br/revistaenfermagem/index.php/revista/ article/viewFile/998/pdf_157

9. Figueiredo PP, Lunardi Filho WD, Silveira RS, Fonseca AD.The non-implementation of the nursing process: reflection based on Deleuze's and Guattari's concepts.
Texto Contexto Enferm [Internet]. 2014 [cited 2016 Set 07]; 23(4). Available from: http://www.scielo.br/pdf/ tce/v23n4/pt_0104-0707-tce-23-04-01136.pdf

10.Silva AS, Nóbrega MML, Macedo WCM. Nursing diagnoses/outcomes for parturient and puerperal women using the International Classification for Nursing Practice. Rev Eletr Enferm [Internet]. 2012 [cited 2016 Set 07]; 14(2):267-76. Available from: https://www.fen.ufg.br/ fen_revista/v14/n2/v14n2a06.htm

11.Nóbrega MML, Garcia TR, Furtado LG, Albuquerque CC, Lima CLH. Nursing terminologies: from the NANDA Taxonomy to International Classification for the Nursing Practice. Rev Enferm UFPE on line. [Internet]. 2008 [cited 2012 Jun 30]; 2(4):390-6. Available from: http://dx.doi. org/10.5205/reuol.333-11493-1-LE.0204200817

12.Silva SL, Marques IS. Análise do registro de dados obstétricos em prontuários. Cogitare Enferm [Internet]. 2007 [cited 2012 Jun 30]; 12(2):150-6. Available from: http:/ / ojs.c3sl.ufpr.br/ojs2/index.php/cogitare/article/view/6937/6727

13. Caixeiro SMO, Dargam B, Thompson GN. Comunicação escrita: importância para os profissionais de enfermagem nas salas de pré-parto. Rev Enferm UERJ [Internet]. 2008 [cited 2012 Jun 30]; 16(2):218-23. Available from: http:/ / www.facenf.uerj.br/v16n2/v16n2a13.pdf

14.Brokel J. Utilização de avaliações de enfermagem, diagnósticos, intervenções e resultados dentro dos prontuários eletrônicos de saúde. In: Herdman TH, organizdor PRONANDA. Programa de atualização em diagnósticos de enfermagem: ciclo 2, v. 4. Porto Alegre (RS): Artmed/ Panamericana; 2014.

15.Martino MMF, Fogaça LF, Costa PCP, Toledo VP. Analysis of the application of nursing process in a governmental hospital. Rev Enferm UFPE on line. [Internet] 2014 [cited 2016 Sep 07]; 8(5):1247-53. Available from: https:/ / periodicos.ufpe.br/revistas/revistaenfermagem/article/view/9806/9974

16.Silva MR, Bittencourt ARC, Diccini S, Balasco A, Barbosa DA. Diagnósticos de enfermagem em portadores da Síndrome da Imunodeficiência Adquirida. Rev Bras Enferm [Internet]. 2009 [cited 2017 May 20]; 62(1):92-9. Available from: http:/ / www.scielo.br/pdf/reben/v62n1/14.pdf

17.Santos RB, Ramos KS. Systematization of nursing care in the Obstetrical Center. Rev Bras Enferm [Internet]. 2012 Dec [cited 2016 Sep 07]; 65(1):13-8. Available from: http:/ / www.scielo.br/scielo.php?script $=$ sci_arttext\&pid= S0034-71672012000100002\&lng=en\&nrm=iso

18. Marian MJS, Tomiolo LV, Moravcik MY. A humanização do cuidado na ótica das equipes da ESF de um município do interior paulista, Brasil. Rev Latino-am Enfermagem [Internet]. 2010 [cited 2017 May 21]; 18(4):763-9. Available from: http://www.scielo.br/pdf/rlae/ v18n4/pt_15.pdf

19. Dantas CN, Santos VEP, Tourinho FSV. Nursing consultation as a technology for care in light of the thoughts of Bacon and Galimberti. Texto Contexto Enferm [Internet]. 2016 [cited 2016 Sep 07]; 25(1). Available from: http:/ / www.scielo.br/pdf/tce/v25n1/en_0104-0707tce-25-01-2800014.pdf

20.Souza Junior DI, Ribeiro JHM, Santos RP, Fagundes KVDL, Dias PF, Mendes MA. Impasses, condições e potencialidades à implementação do processo de enfermagem na prática hospitalar brasileira: revisão integrativa. 
Rev Enferm UFPE on line [Internet]. 2017 [cited 2017 May 20]; 11(2):656-66. Available from: http://www.revista.ufpe.br/revistaenfermagem/index.php/revista/ article/view/8413/pdf_2561

21.Pereira JS, Costa MS, Eloi AC, Araújo BPL, Lima YSM. Introjeção do processo de enfermagem como tecnologia do cuidar em uma instituição hospitalar. Rev Pesq: Cuid Fundam on line. [Internet]. 2013 [cited 2017 May
19]; 5(1):3343-51. Available from: http://www.ssoar. info/ssoar/bitstream/handle/document/32867/ssoarrevpesquisa-2013-1-pereira_et_al-Introjection_of_the_ nursing_process.pdf?sequence $=1$

22.Herdman TH, Kamitsuru S. Perguntas feitas com frequência. In: Nanda-I. Diagnósticos de enfermagem da NANDA International: definições e classificações: 20152017. Porto Alegre (RS): Artmed; 2015. 